\title{
Broadband Bioimpedance Spectroscopy Based on a Multifrequency Mixed Excitation and Nuttall Windowed FFT Algorithm
}

\author{
Yuxiang Yang, ${ }^{1}$ Wen Zhang, ${ }^{1}$ Fangling Du, ${ }^{1}$ Xuan Tang, ${ }^{1}$ He Wen, ${ }^{2}$ and Zhaosheng Teng ${ }^{2}$ \\ ${ }^{1}$ Department of Precision and Instrumentation Engineering, Xian University of Technology, Xian 710048, China \\ ${ }^{2}$ Department of Instrumentation Science and Technology, Hunan University, Changsha 410082, China \\ Correspondence should be addressed to Yuxiang Yang; yyxflyinger@gmail.com
}

Received 27 February 2014; Accepted 19 May 2014; Published 4 June 2014

Academic Editor: Panos Liatsis

Copyright ( $\odot 2014$ Yuxiang Yang et al. This is an open access article distributed under the Creative Commons Attribution License, which permits unrestricted use, distribution, and reproduction in any medium, provided the original work is properly cited.

Bioimpedance spectroscopy (BIS) has become an important clinical indicator for monitoring the pathological status of biological tissues, and multifrequency simultaneous measurement of BIS may provide more accurate diagnostic information compared with the traditional frequency-sweep measurement technology. This paper proposes a BIS multifrequency simultaneous measurement method based on multifrequency mixed (MFM) signal excitation and a Nuttall windowed interpolation FFT algorithm. Firstly, the excitation source adopts the nine-frequency MFM signal $f(9, t)$, which has excellent spectral characteristic and is very suitable for BIS measurement. On this basis, a Nuttall window is adopted to truncate sample data, and an interpolation FFT algorithm based on Nuttall window is built to perform spectral analysis, in which the parameter correction formula is provided based on polynomial approximation. A BIS measurement simulation experiment is performed on an RC three-element equivalent circuit, and results on the 9 primary harmonic frequencies ranging from $3.9 \mathrm{kHz}$ to $1 \mathrm{MHz}$ show a high accuracy with the impedance amplitude relative error $\left|E_{z}\right|<0.3 \%$, and the phase absolute error $\left|E_{p}\right|<0.1^{\circ}$. This paper validates the feasibility of BIS multifrequency simultaneous measurement method and establishes an algorithm foundation for the development of practical broadband BIS measurement system.

\section{Introduction}

Bioimpedance spectroscopy (BIS), which performs measurement of complex electrical impedance of biomaterials over a certain frequency range, has been extensively used in many electrochemical and biomedical applications since it has been shown that BIS can provide physiological and pathological information of biological tissues [1]. In recent years, BIS technology has been increasingly and widely studied and adopted in clinical diagnoses on tissue ischemia [2], mammary cancer [3], and lung cancer [4].

Traditionally, the most commonly used BIS measurement technique is the traditional frequency-sweep (FS) approach which performs measurement of impedance at a single frequency point in a time and sweeps the specific frequencies in the range of interest $[5,6]$. To date, the FS method remains popular for its simplicity and ability to describe the stationary properties of linear time-invariant bioimpedance [7]. The main advantage of the FS approach lies in the fact that it can usually obtain high signal-to-noise ratio (SNR) at the cost of the long measuring time, which usually lasts one second to tens of seconds for a complete sweep $[8,9]$. However, the physiological status of living body, such as the cardiovascular system, is time-varying and dynamic because of its blood flow, heartbeat, and other factors. Therefore, in the situation of time-varying bioimpedance measurement, the main drawback of the FS method is its sweeping cycle, which can be much longer than the bioimpedance time variations [8]. So the BIS data based on the FS measurement technology cannot accurately represent the instantaneous impedance spectra and may lost important diagnostic information [10,11].

In recent years, the multifrequency simultaneous (MFS) measurement technique, which applies a broadband excitation and gains its resulting frequency response by means 
of spectral analysis using the fast Fourier transform (FFT) algorithm, has been becoming popular for time-varying bioimpedance $[8,12-15]$. Thanks to multiple frequencies being excited at the same time, the time to acquire a complete BIS is drastically reduced to tens of milliseconds $[4,16]$. Compared to the FS approach, this MFS approach can obtain the impedance frequency response at different frequencies simultaneously and is highly desirable for time-varying biological systems, such as the respiratory or cardiovascular systems $[17,18]$.

Proper spread-spectrum excitation is crucial for MFS measurement of BIS. Sanchez et al. [15] had a review on several broadband excitations such as maximum length binary sequences (MLBS), chirp. As a binary signal, the MLBS has the advantage of implementation simplicity and has a dense (continuous) amplitude spectrum with plateau envelope where most of the energy is concentrated. But in most BIS applications, only a discrete set of frequencies is required to fit the impedance data to a model like Cole equation [19], and BIS devices usually need several to tens of isolated frequencies (usually with large frequency intervals) over a wide range of frequency (often from $5 \mathrm{kHz}$ to $1 \mathrm{MHz}$ ) [20]. Consequently, broadband excitation with sparse spectrum where energy is distributed on finite frequency points with large frequency spacing is ideal for BIS measurements.

The authors previously proposed a multifrequency mixed (MFM) signal whose majority (65\% or more) of energy is concentrated on several expected 2 nth primary harmonics [21], which provides a proper broadband excitation for MFS measurement BIS. With the broadband excitation, the complex impedance spectrum then can be just calculated as the ratio of the complex Fourier coefficients of the response voltage to the complex Fourier coefficients of the excitation current signal after FFT operations $[15,22]$.

However, FFT operations usually bring spectral leakage since rigorous integer-period sampling is usually hard to achieve. The digital Fourier transform (DFT) theory always assumes that the input sequence is periodic (assumed period extension), so if the input sequence finishes on an integer number of periods everything is fine. Otherwise, discontinuity occurs in the assumed extended sequences and brings spectral leakage and picket fence effect in FFT operation [23, 24]. Unfortunately, integer-period sampling is usually hard to achieve, since sampling device is hard to begin and ends exactly at the signal's head and tail, respectively. Moreover, as a rectangular wave, the MFM signal contains a great number of undesired harmonic components according to the Fourier series theory [25]. These undesired frequency components, especially those whose frequencies are higher than half of the sampling rate, will lead to spectrum aliasing when performing FFT operations and ultimately degrade the measurement precision [26].

Fortunately, the undesired effects of the spectral leakage can be minimized by weighing the samples by a suitable time window, and the picket fence effect can be reduced by adopting interpolation algorithms [27]. It is proved that windows with big side-lobe attenuation and high side-lobe roll-off rate can sufficiently reduce the spectral leakage [28].

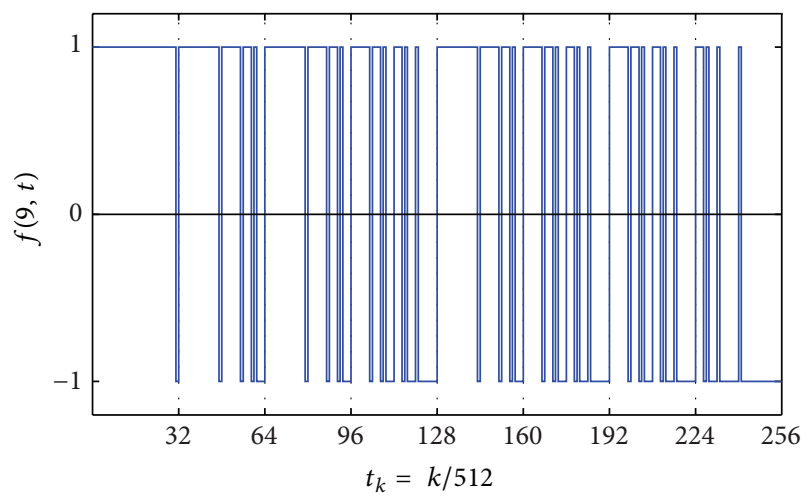

Figure 1: The wave of the MFM signal $f(9, t)$ within a half-period $[0,0.5]$.

This paper synthesizes a nine-frequency MFM signal $f(9, t)$ according to the previously proposed method [21] as the broadband excitation, and realizes multifrequency simultaneous (MFS) measurement of BIS based on a Nuttall windowed interpolation FFT Algorithm. The Nuttall window [29], which has excellent characteristics on side-lobe and main-lobe, is adopted to suppress spectral leakage before FFT, and a Nuttall windowed double-spectral-line interpolation FFT algorithm is developed to reduce the error of picket fence effects. A BIS measurement experiment on an RC three-element equivalent circuit is simulated to evaluate the performance of the proposed algorithm.

\section{Synthesis of the MFM Signal}

According to the MFM signal synthesis principle introduced by the literature [21], let $N=9$; then the nine-frequency MFM signal $f(9, t)$ is synthesized and the time domain waveform of $f(9, t)$ within one period is shown in Figure 1 . The harmonic amplitude spectra and power percentage spectra of $f(9, t)$ are shown in Figures 2(a) and 2(b), respectively, where the nine expected 2nth harmonics (the red solid points in Figure 1), namely, the 1st, 2nd, 4th, 8th, 16th, 32nd, 64th, 128th, and 256th harmonics (we called them primary harmonics thereafter) are obviously prominent. The amplitude spectra $b_{k}$, power spectra $p_{k}$, and initial phase $\varphi_{k}$ of $f(9, t)$ are shown in Table 1 , respectively.

As shown in Figure 2 and Table 1, the synthesized MFM signal $f(9, t)$ has nine 2 nth primary harmonic components which occupy up to $65.52 \%$ of the total power. The period of one code element width of $f(9, t)$ is $0.5 \mu \mathrm{s}$, and its total 512 code elements last a fundamental period $T_{0}=256 \mu \mathrm{s}$, which means that the fundamental frequency of $f(9, t)$ is $f_{0}=3.90625 \mathrm{kHz}$. The nine primary harmonic frequencies of $f(9, t)$ are also listed in Table 1 , which expand from $3.90625 \mathrm{kHz}$ to $1 \mathrm{MHz}$, and cover the main frequency range in most BIS measurements. As a rectangular wave, the MFM signal has a crest factor (CF) of $1.235(1 / \sqrt{0.6552}$, lower than the CF of the sinusoid 1.414) according to the computation method mentioned in [30], which is desirable for BIS of living biological systems because a low crest factor may limit the applied current peaks and set biological systems 
TABLE 1: Spectrum characteristics of the nine primary harmonics in the MFM signal $f(9, t)$.

\begin{tabular}{lccccccccc}
\hline$H_{k}$ & $f_{0}$ & $2 f_{0}$ & $4 f_{0}$ & $8 f_{0}$ & $16 f_{0}$ & $32 f_{0}$ & $64 f_{0}$ & $128 f_{0}$ & $256 f_{0}$ \\
\hline$b_{k}$ & 0.3993 & 0.3988 & 0.3976 & 0.3953 & 0.3909 & 0.3827 & 0.3688 & 0.3482 \\
$p_{k}(\%)$ & 7.97 & 7.95 & 7.90 & 7.81 & 7.64 & 7.32 & 6.80 & 6.06 \\
$\varphi_{k}$ & 0 & 0 & 0 & 0 & 0 & 0 & 0.3482 \\
$f(\mathrm{kHz})$ & 3.90625 & 7.8125 & 15.625 & 31.25 & 62.5 & 125 & 250 & 0 & 500 \\
\hline
\end{tabular}

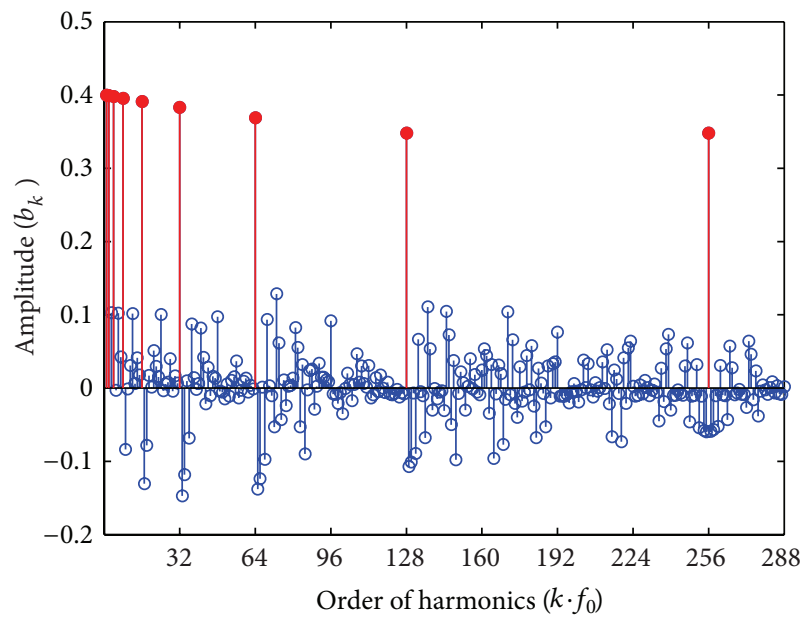

(a) The amplitude spectra of $f(9, t)$

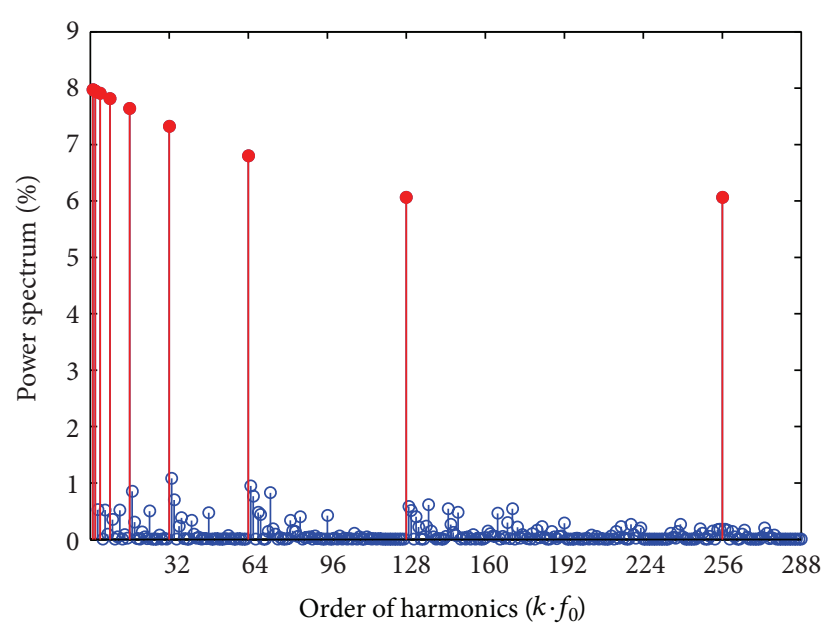

(b) The power spectra of $f(9, t)$

FIgURE 2: The amplitude spectra and power spectra of the MFM signal $f(9, t)$.

within the linear regime $[12,31]$. Furthermore, as a binaryvalued function, the MFM signal can be generated by FPGA easily, and the bandwidth of its harmonics can be variable by changing the FPGA's operating clock, and the number of the expected primary harmonics is also adjustable. Based on the features mentioned above, the newly designed wideband excitation source establishes a good foundation for fast measurement of BIS.

\section{Nuttall Windowed Interpolation FFT Algorithm}

3.1. Nuttall Window. The Nuttall window [29] is a combination of cosine windows and can be expressed in the time domain as follows:

$$
w_{N}(n)=\sum_{m=0}^{M-1}(-1)^{m} b_{m} \cos \left(2 \pi n \cdot \frac{m}{N}\right),
$$

where $M$ is the number of terms of the cosine function; $N$ denotes the length of the window and $n=0,1, \ldots, N-$ 1 ; the coefficient $b_{m}$ should meet the constraint conditions: $\sum_{m=0}^{M-1} b_{m}=1, \sum_{m=0}^{M-1}(-1)^{m} b_{m}=0$.

The coefficient groups of typical Nuttall windows shown in Tables 2 and 3 list the side-lobe characteristics of typical Nuttall windows and other cosine-combined windows [29].

The side-lobe attenuation level and asymptotic decay rate directly affect the FFT-based spectral analysis results [28]. Among the windows shown in Table 2, the 4-term 3-order
Nuttall window has the fastest side-lobe asymptotic decay rate $30 \mathrm{~dB} /$ oct and an acceptable side-lobe attenuation level $-82.6 \mathrm{~dB}$, which is adopted to truncate sampled data and suppress spectrum leakage in FFT.

3.2. Nuttall Windowed Interpolation FFT Algorithm. If $x(t)$ denotes an analog signal with multiple harmonic components, its discrete-time form $x(n)$ can be expressed as

$$
x(n)=\sum_{h=1}^{H} A_{h} \sin \left(2 \pi \frac{h f_{1}}{f_{s}} n+\varphi_{h}\right),
$$

where $H$ denotes the number of harmonic components and $h$ is an integer representing the harmonic order. When $h=1$, $f_{1}, A_{1}, \varphi_{1}$ are the frequency, amplitude, and initial phase of the fundamental harmonic, respectively. When $h \neq 1, h f_{1}, A_{h}$, $\varphi_{h}$ denote the frequency, amplitude, and initial phase of the $h$ th harmonic respectively. $f_{s}$ is the digital sampling rate.

If $x(n)$ is truncated by Nuttall window $w_{N}(n)$, then the windowed signal after discrete Fourier transform (DFT) can be expressed in frequency domain as

$$
\begin{aligned}
X(k \Delta f) & =\sum_{h=1}^{H} \frac{A_{h}}{2 j} e^{j \varphi_{h}} W_{N}\left(\frac{2 \pi\left(k \Delta f-h f_{1}\right)}{f_{s}}\right) \\
& =\sum_{h=1}^{H} \frac{A_{h}}{2 j} e^{j \varphi_{h}} W_{N}\left(\frac{2 \pi\left(k-k_{h}\right)}{N}\right),
\end{aligned}
$$

where $W_{N}(f)$ denotes the continuous spectrum of $w_{N}(n), \Delta f$ the frequency resolution, and $\Delta f=f_{s} / N$. $N$ is the truncated 
TABLE 2: Coefficient groups of typical Nuttall windows.

\begin{tabular}{lcccc}
\hline Coefficients & 3-order minimum side-lobe & 4-order minimum side-lobe & 4-term 1-order & 4-term 3-order \\
\hline$b_{0}$ & 0.4243801 & 0.3635819 & 0.355768 & 0.338946 \\
$b_{1}$ & 0.4973406 & 0.4891775 & 0.487396 & 0.481973 \\
$b_{2}$ & 0.0782793 & 0.1365995 & 0.144232 & 0.161054 \\
$b_{3}$ & & 0.0106411 & 0.012604 & 0.018027 \\
\hline
\end{tabular}

TABLE 3: Side-lobe characteristics of Nuttall windows and other cosine-combined windows.

\begin{tabular}{lcc}
\hline Types of windows & Peak side-lobe (dB) & Asymptotical decay (dB/oct) \\
\hline Hann. window & -32 & 18 \\
Blackman window & -58 & 18 \\
Blackman-Harris window & -92 & 6 \\
3-order Nuttall window with minimum side-lobe & -71.49 & 6 \\
4-order Nuttall window with minimum side-lobe & -98.2 & 6 \\
4-term 1-order Nuttall window & -93.3 & 18 \\
4-term 3-order Nuttall window & -82.6 & 30 \\
\hline
\end{tabular}

data length. $h f_{1}$ is the peak spectral of the $h$ th harmonic and $h f_{1}=k_{h} \Delta f$.

When signal is sampled asynchronously, its peak frequency of the $h$ th harmonic $k_{h} \Delta f$ can hardly be exactly on the discrete spectral line. As shown in Figure 3, suppose that the largest and the second largest spectral amplitude lines near the peak point $k_{h}$ are $k_{h 1}$ and $k_{h 2}$, respectively; they have the relationship

$$
k_{h 1} \leq k_{h} \leq k_{h 2}=k_{h 1}+1
$$

And the amplitudes of these two spectral lines, $y_{1}$ and $y_{2}$, can be expressed as

$$
\begin{aligned}
y_{1} & =\left|X\left(k_{h 1} \Delta f\right)\right|=\left|\frac{A_{h}}{2 j} e^{j \varphi_{h}} W_{N}\left(\frac{2 \pi\left(k_{h 1}-k_{h}\right)}{N}\right)\right| \\
& =\frac{A_{h}}{2}\left|W_{N}\left(\frac{2 \pi\left(k_{h 1}-k_{h}\right)}{N}\right)\right|, \\
y_{2}= & \left|X\left(k_{h 2} \Delta f\right)\right|=\left|\frac{A_{h}}{2 j} e^{j \varphi_{h}} W_{N}\left(\frac{2 \pi\left(k_{h 2}-k_{h}\right)}{N}\right)\right| \\
= & \frac{A_{h}}{2}\left|W_{N}\left(\frac{2 \pi\left(k_{h 2}-k_{h}\right)}{N}\right)\right| .
\end{aligned}
$$

Set

$$
\beta=\frac{y_{2}-y_{1}}{y_{2}+y_{1}}
$$

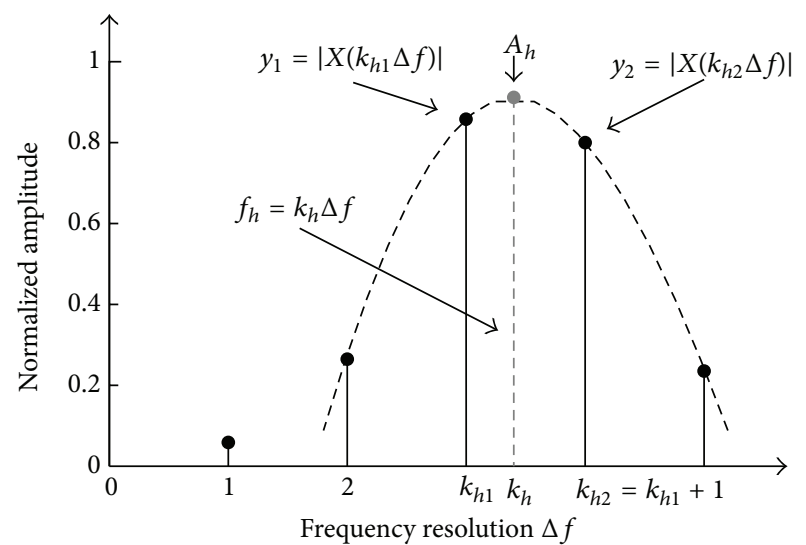

FIGURE 3: Amplitude spectrum of the $h$ th harmonic of $x(n)$ by asynchronous sampling.

And according to (4), set

$$
\begin{aligned}
& k_{h 1}-k_{h}=-\alpha-0.5, \\
& k_{h 2}-k_{h}=-\alpha+0.5 .
\end{aligned}
$$

Then $\alpha$ is in the range $[-0.5,0.5]$ and $\beta$ can be expressed as

$$
\beta=\frac{\left|W_{N}(2 \pi(-\alpha+0.5) / N)\right|-\left|W_{N}(2 \pi(-\alpha-0.5) / N)\right|}{\left|W_{N}(2 \pi(-\alpha+0.5) / N)\right|+\left|W_{N}(2 \pi(-\alpha-0.5) / N)\right|} .
$$

Let $\alpha$ be the inverse function of (8); namely, $\alpha=u^{-1}(\beta)$; then the frequency $f_{h}$ can be corrected by $\alpha$ :

$$
f_{h}=k_{h} \Delta f=\left(\alpha+k_{h 1}+0.5\right) \Delta f .
$$


And set the amplitude $A_{h}$ as the weighted average of the amplitudes of the $k_{h 1}$ and $k_{h 2}$ spectral lines:

$$
\begin{aligned}
A_{h}= & \left(A_{h 2}\left|W_{N}\left(2 \pi\left(k_{h 2}-k_{h}\right) / N\right)\right|\right. \\
& \left.+A_{h 1}\left|W_{N}\left(2 \pi\left(k_{h 1}-k_{h}\right) / N\right)\right|\right) \\
& \times\left(\left|W_{N}\left(2 \pi\left(k_{h 2}-k_{h}\right) / N\right)\right|\right. \\
& \left.+\left|W_{N}\left(2 \pi\left(k_{h 1}-k_{h}\right) / N\right)\right|\right)^{-1} \\
= & 2\left(y_{1}+y_{2}\right) \\
& \times\left(\left|W_{N}(2 \pi(-\alpha+0.5) / N)\right|\right. \\
& \left.+\left|W_{N}(2 \pi(-\alpha-0.5) / N)\right|\right)^{-1} .
\end{aligned}
$$

According to (3) and (7), the initial phase of the $h$ th harmonic can be corrected as follows:

$$
\begin{aligned}
\varphi_{h} & =\arg \left[X\left(k_{h 1} \Delta f\right)\right]+\frac{\pi}{2}-\arg \left[W_{N}\left(\frac{2 \pi\left(k_{h 1}-k_{h}\right)}{N}\right)\right] \\
& =\arg \left[X\left(k_{h 1} \Delta f\right)\right]+\frac{\pi}{2}-\arg \left[W_{N}\left(\frac{2 \pi(-\alpha-0.5)}{N}\right)\right] .
\end{aligned}
$$

According to (1), the spectrum amplitude function of Nuttall window is

$$
\begin{aligned}
& W_{N}(w) \\
& =\sum_{m=0}^{M-1}(-1)^{m} \frac{b_{m}}{2}\left[W_{R}\left(w-\frac{2 \pi}{N} m\right)+W_{R}\left(w+\frac{2 \pi}{N} m\right)\right],
\end{aligned}
$$

where $W_{R}(w)$ denotes the spectrum function of rectangular window and $W_{R}(w)=(\sin (N w / 2) / \sin (w / 2)) e^{-j((N-1) / 2) w}$.

Let $w=(2 \pi / N) k$; then (12) can be expressed as

$$
\begin{aligned}
W_{N}\left(\frac{2 \pi}{N} k\right)= & \sin \pi k \cdot e^{-j \pi k} \\
\times & {\left[\sum_{m=0}^{M-1}(-1)^{m} \frac{b_{m}}{2}\right.} \\
& \left.\cdot \frac{\sin (2 \pi k / N)}{\sin (\pi(k-m) / N) \sin (\pi(k+m) / N)}\right] .
\end{aligned}
$$

Because $|-\alpha \pm 0.5| \leq 1, k=-\alpha \pm 0.5$, and $N$ is generally very large, (13) can be approximately expressed as

$$
\begin{aligned}
& \left|W_{N}\left(\frac{2 \pi(-\alpha \pm 0.5)}{N}\right)\right| \\
& \quad \approx \mid \sin \pi(-\alpha \pm 0.5) \cdot\left[\sum_{m=0}^{M-1}(-1)^{m} \frac{b_{m}}{\pi} \frac{N(-\alpha \pm 0.5)}{(-\alpha \pm 0.5)^{2}-m^{2}}\right] .
\end{aligned}
$$

Substituting (14) into (8), we can obtain the approximation of $\alpha$ :

$$
\alpha=u^{-1}(\beta)=H(\beta)
$$

Similarly, substituting (14) into (10), we can obtain the approximation of (10):

$$
A_{h}=\frac{\left(y_{1}+y_{2}\right)}{N} v(\alpha)
$$

where $v(\alpha)$ denotes a function of $\alpha$ and can be expressed as follows:

$$
v(\alpha)=\frac{2 N}{\left|W_{N}(2 \pi(-\alpha+0.5) / N)\right|+\left|W_{N}(2 \pi(-\alpha-0.5) / N)\right|} .
$$

From (11) and (14), the phase can be corrected as follows:

$$
\varphi_{h}=\arg \left[X\left(k_{h 1} \Delta f\right)\right]+\frac{\pi}{2}-\pi(-\alpha-0.5) .
$$

As shown in (9), (16), and (18), $f_{h}, A_{h}, \varphi_{h}$ are all related to $\alpha$, but $\alpha$ has a complicated relationship with $\beta$ in (8) and with $v(\alpha)$ in (17). However, the value of $\alpha$ and $v(\alpha)$ could hardly be figured out by direct analytical method.

This paper proposes a simple and efficient method based on polynomial approximation to estimate the values of $\alpha$ and $v(\alpha)$. Let $\alpha$ be a series of values in the range $[-0.5,0.5]$; then the corresponding values of $\beta$ and $v(\alpha)$ can be computed according to (14), (8), and (17). Afterwards, based on the known mapping relationship between $\alpha$ and $\beta$, the inverse function of $\beta$, namely, $\alpha=u^{-1}(\beta)$, can be approximated by a quintic polynomial of $\beta$ :

$$
\begin{aligned}
\alpha= & u^{-1}(\beta)=H(\beta)=2.95494514 \beta \\
& +0.17671943 \beta^{3}+0.09230694 \beta^{5}
\end{aligned}
$$

Also, based on the known mapping relationship between $\alpha$ and $v(\alpha), v(\alpha)$ can be approximated by a quintic polynomial of $\alpha$ :

$$
v(\alpha)=3.20976143+0.9187393 \alpha^{2}+0.14734229 \alpha^{4} .
$$

For every harmonic, we always can find the largest and the second largest spectral lines $k_{h 1}$ and $k_{h 2}$ and their amplitudes $y_{1}$ and $y_{2}$. Then $\beta$ must have a concrete value according to (6), and the values of $\alpha$ and consequent $v(\alpha)$ can be calculated by (19) and (20), respectively. With the determined $\alpha$ and $v(\alpha)$, the frequency, amplitude, and initial phase of the $h$ th harmonic, $f_{h}, A_{h}, \varphi_{h}$, are corrected according to (9), (16), and (18).

As to the MFM signal $f(9, t)$, which contains multiple harmonic components, set $h=1,2,4,8,16,32,64,128$, and 256 , and the corresponding primary harmonic parameters $f_{h}$, $A_{h}, \varphi_{h}$, can be computed, respectively. 


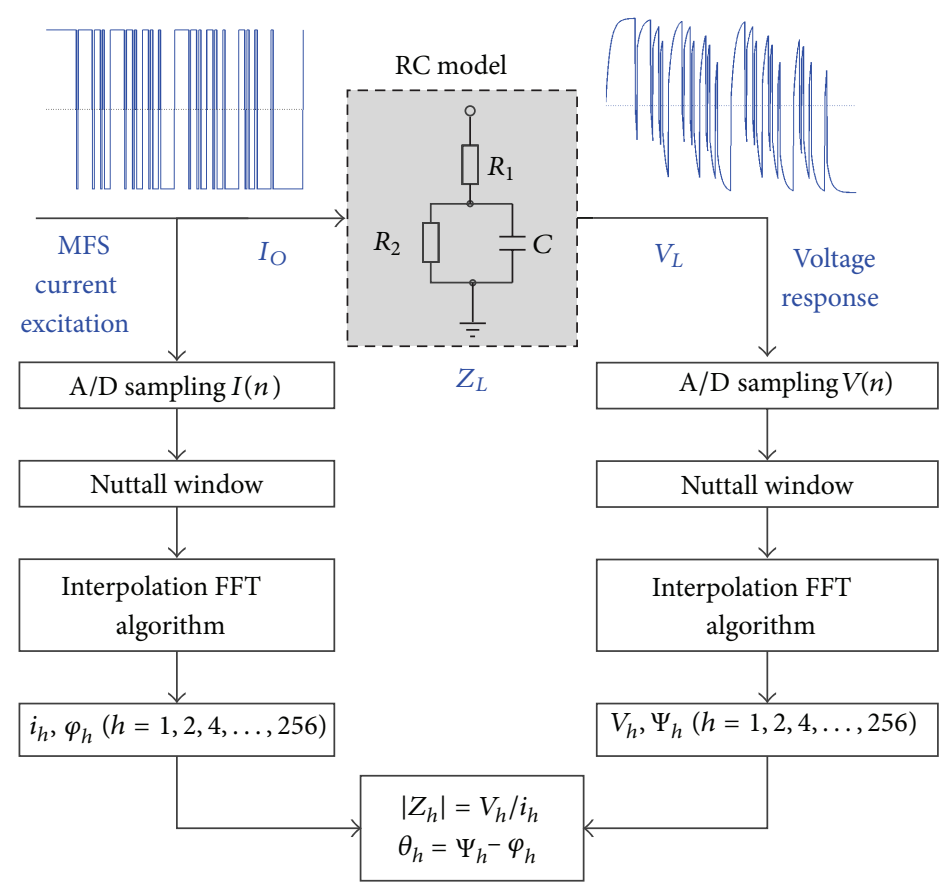

FIGURE 4: BIS multifrequency synchronous measurement scheme.

\section{Measurement Experiment}

On the basis of Nuttall windowed interpolation FFT algorithm mentioned above, we can establish a BIS multifrequency synchronous measurement scheme as shown in Figure 4.

In Figure 4, the excitation current source $I_{O}$ is the MFM signal $f(9, t), I_{O}$ flows through the load $Z_{L}$, and the voltage drop $V_{L}$ occurs. In biological tissue, the extracellular and intracellular fluids can be simply equivalent to a resistance, and the cell membrane can be equivalent to a capacitance, which supports the hypothesis that an $\mathrm{RC}$ three-element model can represent equivalently the impedance of a biological tissue [1]. This paper adopts an RC three-element equivalent circuit model (dashed box in Figure 4) as the load $Z_{L}$. In this experiment, we use the typical parameters $R_{1}=$ $330 \Omega, R_{2}=590 \Omega$, and $C=4.7 \mathrm{nF}$, since the human body impedance range is $300 \sim 1500 \Omega$, the capacitance value range is $2 \mathrm{pF} \sim 22 \mathrm{nF}$ [32].

In order to prove the correctness of the scheme proposed above, we design a BIS measurement simulation experiment. When the RC three-element equivalent circuit is driven by the periodical current source signal $f(9, t)$, the response voltage signal $V_{L}$ at any time can be simulated according to the method mentioned in the literature [21]. The simultaneous samplings on $I_{O}$ and $V_{L}$ are simulated and the corresponding discrete sequence $I(n)$ and $V(n)$ are obtained, respectively. Figure 5(a) shows the simulated $V_{L}$ in a half-period, and Figure 5(b) gives an example of the simultaneous sampling of the excitation $f(9, t)$ and the response $V_{L}(t)$.

Like most broadband BIS measurements using periodic excitations, the complex impedance spectrum of the tested
RC model can be just calculated as the division of the voltage and current complex Fourier coefficients obtained from their respective FFT operations $[15,22] . I(n)$ and $V(n)$ are firstly truncated using the Nuttall window; then the Nuttall windowed interpolation FFT algorithm is performed on the two sequences, respectively. Since rigorous integer-period sampling is hard to achieve in practice, ten or more sampling cycles are usually needed for the windowed FFT algorithm. In this paper, we complete the sampling in about ten cycles, which lasts about 2.56 milliseconds (the period of $f(9, t) T_{0}=$ $256 \mu \mathrm{s})$. The complex Fourier coefficients of $I(n)$ and $V(n)$, namely, $\left(i_{h}, \varphi_{h}\right)$ and $\left(V_{h}, \Psi_{h}\right)(h=1,2,4,8, \ldots, 256)$ are obtained after FFT. Finally, the tested impedance amplitudes and phases are calculated according to the following formula:

$$
\begin{gathered}
\left|Z_{h}\right|=\frac{\left|V_{h}\right|}{\left|i_{h}\right|}, \\
\theta_{h}=\Psi_{h}-\varphi_{h} .
\end{gathered}
$$

The BIS measurement results at the nine primary harmonics are shown in Table 4, in which the impedance amplitude relative error $\left|E_{z}\right| \leq 0.3 \%$, and the phase absolute error $\left|E_{p}\right|<0.1^{\circ}$.

\section{Conclusion}

Multifrequency simultaneous (MFS) measurement of BIS can greatly reduce measurement time and grasp the transient physiological status of living body compared with the traditional frequency-sweep measurement technology. This paper proposes a BIS multifrequency simultaneous measurement 
TABLE 4: Experiment results of BIS measurement under the excitation of the MFM signal $f(9, t)$.

\begin{tabular}{|c|c|c|c|c|c|c|c|}
\hline Primary harmonic & $\begin{array}{c}\text { Frequency } \\
(\mathrm{kHz})\end{array}$ & $\begin{array}{c}\text { Standard } Z \\
Z s \\
\end{array}$ & $\begin{array}{c}\text { Measured } Z \\
Z_{m} \\
\end{array}$ & $\begin{array}{c}\text { Relative errors } \\
\left|E_{z}\right|(\%)\end{array}$ & Standard Ps & Measured Pm & $\begin{array}{c}\text { Absolute errors }\left|E_{p}\right| \\
\left({ }^{\circ}\right)\end{array}$ \\
\hline$\overline{f_{0}}$ & 3.90625 & 918.1501 & 918.1311 & 0.0021 & -2.4951 & -2.5098 & 0.0148 \\
\hline $2 f_{0}$ & 7.8125 & 912.6795 & 912.8680 & 0.0207 & -4.9562 & -4.9745 & 0.0183 \\
\hline $4 f_{0}$ & 15.625 & 891.9151 & 891.8473 & 0.0076 & -9.6518 & -9.6461 & 0.0057 \\
\hline $8 f_{0}$ & 31.25 & 823.2610 & 822.8110 & 0.0547 & -17.5164 & -17.5659 & 0.0495 \\
\hline $16 f_{0}$ & 62.5 & 668.0581 & 667.5680 & 0.0734 & -26.1026 & -26.1476 & 0.0450 \\
\hline $32 f_{0}$ & 125 & 487.1455 & 486.5256 & 0.1273 & -27.3403 & -27.4284 & 0.0881 \\
\hline $64 f_{0}$ & 250 & 381.8698 & 381.0998 & 0.2016 & -19.6909 & -19.7728 & 0.0819 \\
\hline $128 f_{0}$ & 500 & 344.2257 & 343.3310 & 0.2599 & -11.1973 & -11.2497 & 0.0524 \\
\hline $256 f_{0}$ & 1000 & 333.6487 & 332.7088 & 0.2817 & -5.8059 & -5.8323 & 0.0263 \\
\hline
\end{tabular}

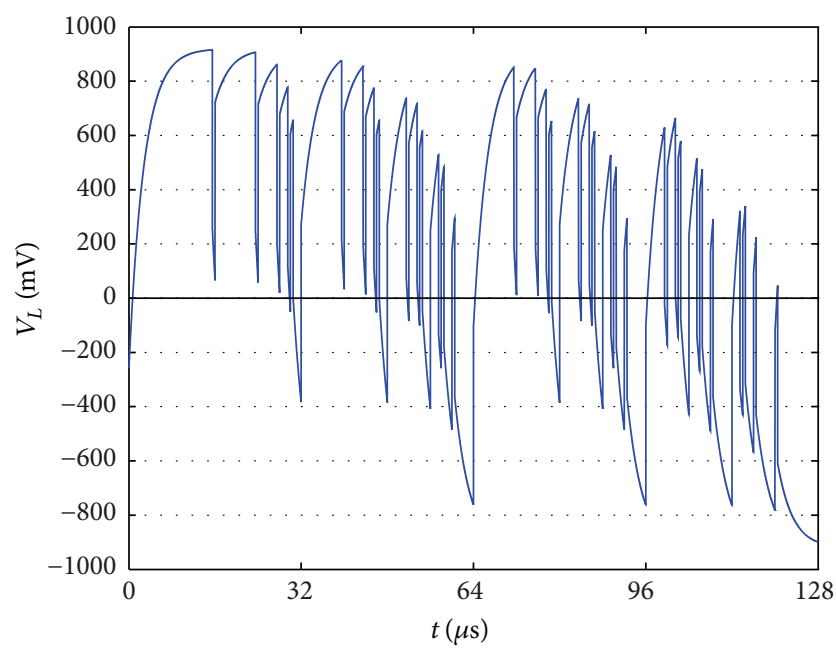

(a) The simulated voltage response $V_{L}(t)$ on the RC three-element load in a half-period
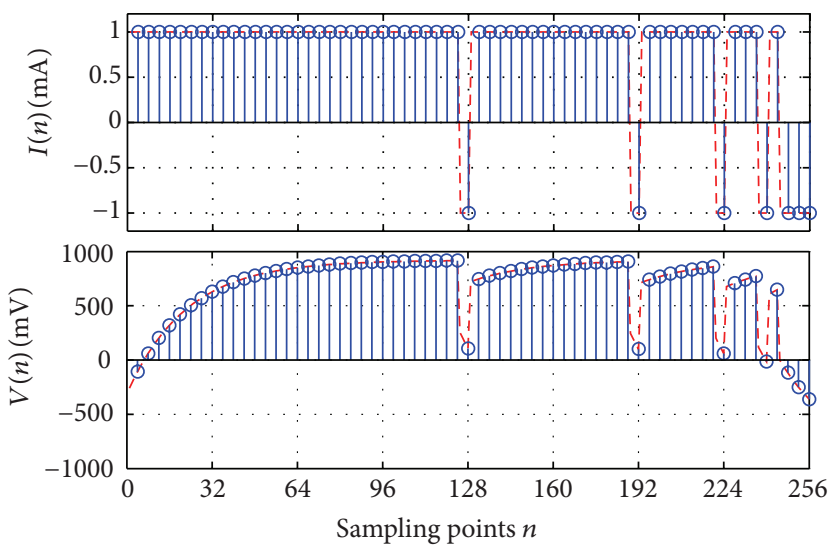

(b) Simultaneous sampling of the excitation $f(9, t)$ and the response $V_{L}(t)$

FIGURE 5: The simulated voltage response $V_{L}(t)$ and simultaneous sampling of the excitation $f(9, t)$ and the response $V_{L}(t)$.

approach based on an MFM excitation and a Nuttall Windowed interpolation FFT algorithm. On the basis of the ninefrequency MFM signal $f(9, t)$ excitation, a Nuttall window is adopted to truncate the sample data, and an interpolation FFT algorithm based on Nuttall window is developed to perform spectral analysis, in which the parameter correction formula is provided based on polynomial approximation. A BIS measurement simulation experiment is performed on a RC three-element equivalent circuit, and results show a high accuracy with the impedance amplitude relative error 
$\left|E_{z}\right| \leq 0.3 \%$, and the phase absolute error $\left|E_{p}\right|<0.1^{\circ}$. This paper establishes an algorithm foundation for development of practical broadband BIS measurement systems.

\section{Conflict of Interests}

The authors declare that they have no conflict of interests.

\section{Acknowledgments}

This study has been partly supported by two grants from the National Natural Science Foundation of China (30900317, 61273271), a grant from the Scientific Research Plan of Education Bureau of Shaanxi Province, China (12JK0527), and a grant from China Postdoctoral Science Foundation (20110491674).

\section{References}

[1] S. Grimnes and G. Ø. Martinsen, Bioimpedance and Bioelectricity Basics, Elsevier Academic Press, London, UK, 2nd edition, 2008.

[2] F. Mellert, K. Winkler, C. Schneider et al., "Detection of (Reversible) myocardial ischemic injury by means of electrical bioimpedance," IEEE Transactions on Biomedical Engineering, vol. 58, no. 6, pp. 1511-1518, 2011.

[3] S. A. Czerniec, L. C. Ward, M. J. Lee, K. M. Refshauge, J. Beith, and S. L. Kilbreath, "Segmental measurement of breast cancerrelated arm lymphoedema using perometry and bioimpedance spectroscopy," Supportive Care in Cancer, vol. 19, no. 5, pp. 703710, 2011.

[4] B. Sanchez, G. Vandersteen, I. Martin et al., "In vivo electrical bioimpedance characterization of human lung tissue during the bronchoscopy procedure. A feasibility study," Medical Engineering and Physics, vol. 35, pp. 949-957, 2013.

[5] Y. Yang, J. Wang, G. Yu, F. Niu, and P. He, "Design and preliminary evaluation of a portable device for the measurement of bioimpedance spectroscopy," Physiological Measurement, vol. 27, no. 12, pp. 1293-1310, 2006.

[6] U. Pliquett and A. Barthel, "Interfacing the AD5933 for bioimpedance measurements with front ends providing galvanostatic or potentiostatic excitation," Journal of Physics: Conference Series, vol. 407, no. 1, Article ID 012019, 2012.

[7] M. T. Wilson, M. Elbohouty, L. J. Voss, and D. A. Steyn-Ross, "Electrical impedance of mouse brain cortex in vitro from $4.7 \mathrm{kHz}$ to $2.0 \mathrm{MHz}$," Physiological Measurement, vol. 35, article 267, 2014.

[8] B. Sanchez, E. Louarroudi, E. Jorge, J. Cinca, R. Bragos, and R. Pintelon, "A new measuring and identification approach for time-varying bioimpedance using multisine electrical impedance spectroscopy," Physiological Measurement, vol. 34, no. 3, pp. 339-357, 2013.

[9] B. Sanchez, R. Bragos, and G. Vandersteen, "Influence of the multisine excitation amplitude design for biomedical applications using Impedance Spectroscopy," in Proceedings of the Annual International Conference of the IEEE Engineering in Medicine and Biology Society, pp. 3975-3978, 2011.

[10] W. Chao, H. Chunyan, Z. Xiaoli, and W. Huaxiang, "Mixing frequency bio-impedance measurement technology based on DFT and virtual reference vector," in Proceedings of the International
Conference on BioMedical Engineering and Informatics, pp. 455459, May 2008.

[11] M. Min, U. Pliquett, T. Nacke, A. Barthel, P. Annus, and R. Land, "Broadband excitation for short-time impedance spectroscopy," Physiological Measurement, vol. 29, no. 6, pp. S185-S192, 2008.

[12] S. Gawad, T. Sun, N. G. Green, and H. Morgan, "Impedance spectroscopy using maximum length sequences: application to single cell analysis," Review of Scientific Instruments, vol. 78, no. 5, Article ID 054301, 2007.

[13] B. Sanchez, C. R. Rojas, G. Vandersteen, R. Bragos, and J. Schoukens, "On the calculation of the D-optimal multisine excitation power spectrum for broadband impedance spectroscopy measurements," Measurement Science and Technology, vol. 23, no. 8, Article ID 085702, 2012.

[14] B. Sanchez, G. Vandersteen, R. Bragos, and J. Schoukens, "Optimal multisine excitation design for broadband electrical impedance spectroscopy," Measurement Science and Technology, vol. 22, no. 11, Article ID 115601, 2011.

[15] B. Sanchez, G. Vandersteen, R. Bragos, and J. Schoukens, "Basics of broadband impedance spectroscopy measurements using periodic excitations," Measurement Science and Technology, vol. 23, no. 10, Article ID 105501, 2012.

[16] B. Sanchez, A. S. Bandarenka, G. Vandersteen, J. Schoukens, and R. Bragos, "Novel approach of processing electrical bioimpedance data using differential impedance analysis," Medical Engineering and Physics, vol. 35, no. 9, pp. 1349-1357, 2013.

[17] B. Sanchez, J. Schoukens, R. Bragos, and G. Vandersteen, "Novel estimation of the electrical bioimpedance using the local polynomial method. Application to in vivo real-time myocardium tissue impedance characterization during the cardiac cycle," IEEE Transactions on Biomedical Engineering, vol. 58, no. 12, pp. 3376-3385, 2011.

[18] R. L. R. Gordon, M. Min, T. Parve, and R. W. Salo, "A virtual system for simultaneous multi-frequency measurement of electrical bioimpedance," International Journal of Bioelectromagnetism, vol. 7, pp. 243-246, 2005.

[19] F. Seoane, L. C. Ward, K. Lindecrantz, and B. E. Lingwood, "Automated criterion-based analysis for Cole parameters assessment from cerebral neonatal electrical bioimpedance spectroscopy measurements," Physiological Measurement, vol. 33, no. 8, pp. 1363-1377, 2012.

[20] L. C. Ward, T. Essex, and B. H. Cornish, "Determination of Cole parameters in multiple frequency bioelectrical impedance analysis using only the measurement of impedances," Physiological Measurement, vol. 27, no. 9, article no. 007, pp. 839-850, 2006.

[21] Y. Yang, M. Kang, Y. Lu, J. Wang, J. Yue, and Z. Gao, "Design of a wideband excitation source for fast bioimpedance spectroscopy," Measurement Science and Technology, vol. 22, no. 1, Article ID 013001, 2011.

[22] M. Nahvi and B. S. Hoyle, "Electrical impedance spectroscopy sensing for industrial processes," IEEE Sensors Journal, vol. 9, no. 12, pp. 1808-1816, 2009.

[23] H. Ahmad, M. A. Salam, L. Y. Ying, and N. Bashir, "Harmonic components of leakage current as a diagnostic tool to study the aging of insulators," Journal of Electrostatics, vol. 66, no. 3-4, pp. 156-164, 2008.

[24] Y. F. Li and K. F. Chen, "Eliminating the picket fence effect of the fast Fourier transform," Computer Physics Communications, vol. 178, no. 7, pp. 486-491, 2008.

[25] A. V. Oppenheim, A. S. Willsky, and S. H. Nawab, Signals \& Systems, Prentice-Hall, Upper Saddle River, NJ, USA, 2nd edition, 1996. 
[26] A. V. Oppenheim, R. W. Schafer, and J. R. Buck, Discrete-Time Signal Processing, Prentice Hall, Upper Saddle River, NJ, USA, 1999.

[27] G. Andria, M. Savino, and A. Trotta, "Windows and interpolation algorithms to improve electrical measurement accuracy," IEEE Transactions on Instrumentation and Measurement, vol. 38, no. 4, pp. 856-863, 1989.

[28] H. Wen, Z. Teng, and S. Guo, "Triangular self-convolution window with desirable sidelobe behaviors for harmonic analysis of power system," IEEE Transactions on Instrumentation and Measurement, vol. 59, no. 3, pp. 543-552, 2010.

[29] A. H. Nuttall, "Some windows with very good sidelobe behavior," IEEE Transactions on Acoustics, Speech, and Signal Processing, vol. 29, no. 1, pp. 84-91, 1981.

[30] R. Pintelon and J. Schoukens, System Identification: A Frequency Domain Approach, John Wiley \& Sons, New York, NY, USA, 2nd edition, 2012.

[31] T. Paavle, M. Min, P. Annus, A. Birjukov, R. Land, and T. Parve, "Wideband object identification with rectangular wave chirp excitation," in Proceedings of the European Conference on Circuit Theory and Design Conference Program (ECCTD '09), pp. 421424, August 2009.

[32] S. Armstrong and D. Jennings, "Current injection electrodes for electrical impedance tomography," Physiological Measurement, vol. 25, no. 4, pp. 797-802, 2004. 


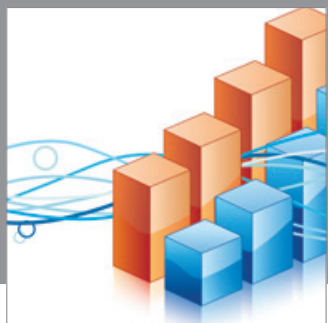

Advances in

Operations Research

mansans

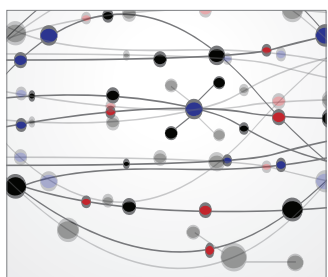

The Scientific World Journal
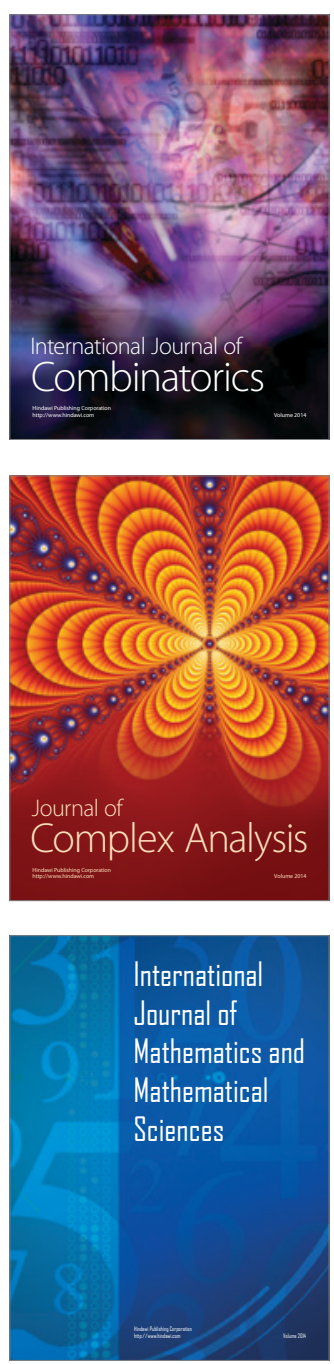
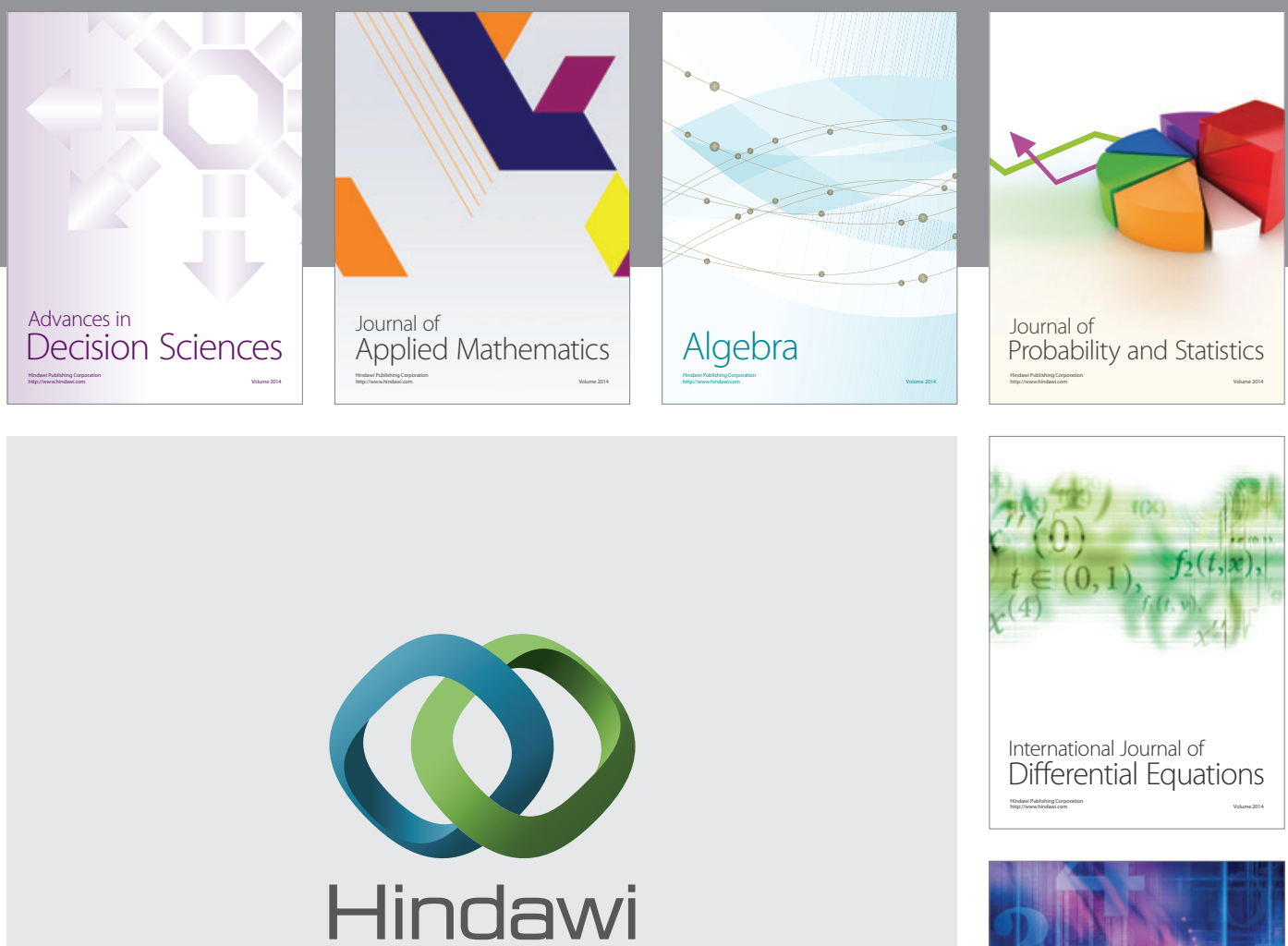

Submit your manuscripts at http://www.hindawi.com
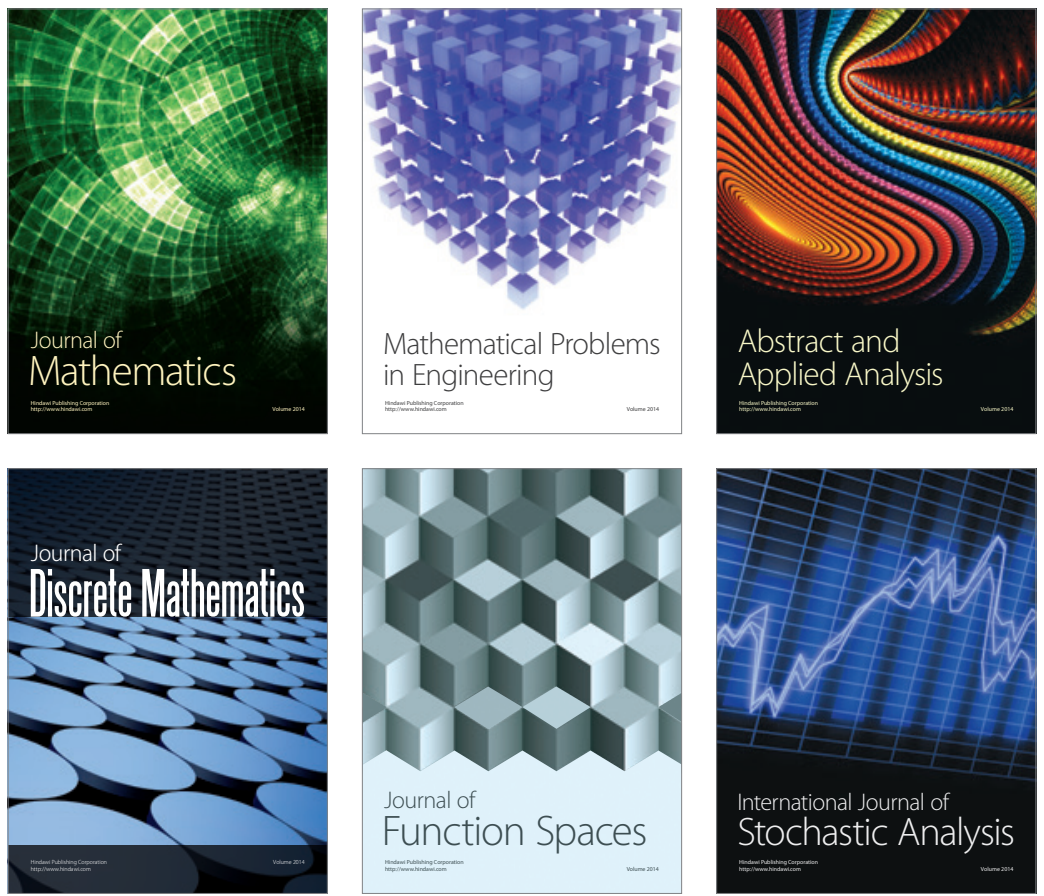

Journal of

Function Spaces

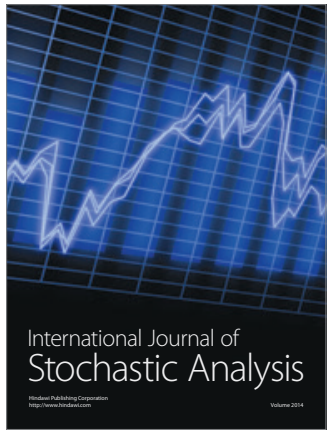

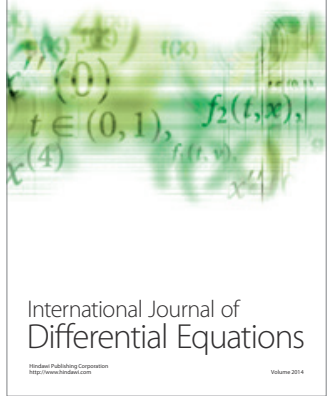
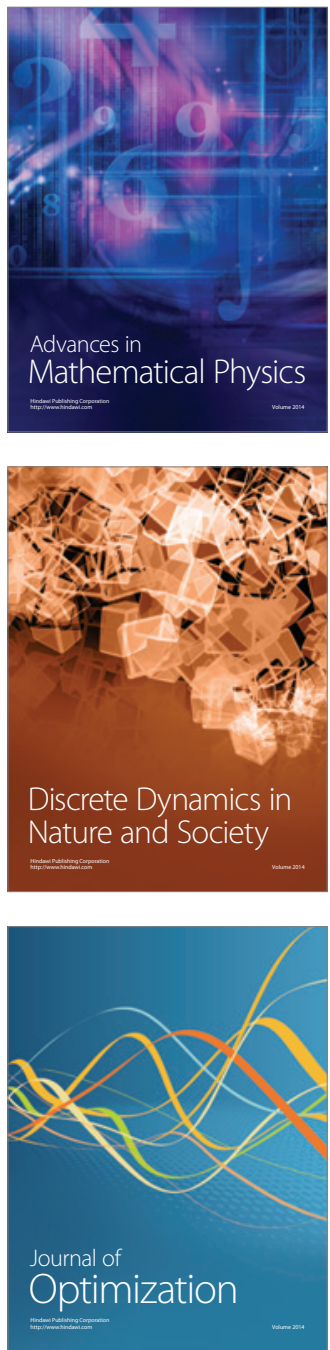